\title{
A Study of Unsteady Rotating Hydromagnetic Free and Forced Convection in a Channel Subject to Forced Oscillation under an Oblique Magnetic Field
}

\author{
S. K. Ghosh ${ }^{1 \dagger}$, O. Anwar Bég ${ }^{2}$ and M. Narahari ${ }^{3}$ \\ ${ }^{1}$ Magnetohydrodynamics Research, Department of Mathematics, Narajole Raj College, P.O.- Narajole, Dist. - \\ Midnapore (West), Pin code - 721 211, West Bengal, India. \\ 2 Biomechanics and Astronautics Research, Aerospace Engineering Program, Mechanical Engineering Subject \\ Group, Department of Engineering and Mathematics, Sheffield Hallam University, Sheffield, S1 1WB, UK. \\ ${ }^{3}$ Fluid Dynamics Research, Fundamental and Applied Sciences Department, Universiti Teknologi Petronas, 31750 \\ Tronoh, Bandar Seri Iskandar, Perak Darul Ridzuan, Malaysia.
}

$\dagger$ †orresponding Author Email: g_swapan2002@yahoo.com

(Received May 20, 2011; accepted October 9, 2011)

\begin{abstract}
A theoretical analysis is presented for transient, fully-developed magnetohydrodynamic free and forced convection flow of a viscous, incompressible, Newtonian fluid in a rotating horizontal parallel-plate channel subjected to a uniform strength, static, oblique magnetic field acting at an angle $\theta$ to the positive direction of the axis of rotation. A constant pressure gradient is imposed along the longitudinal axis of the channel. Magnetic Reynolds number is sufficiently small to negate the effects of magnetic induction. The channel plates are electrically non-conducting. The conservation equations are formulated in an $(x, y, z)$ coordinate system and normalized using appropriate transformations. The resulting non-dimensional coupled ordinary differential equations for primary and secondary velocity components and transformed boundary conditions are found to be reciprocal of the Ekman number $\left(K^{2}=\right.$ 1/Ek), non-dimensional pressure gradient parameter (Px), Hartmann number $\left(M^{2}\right)$, Grashof number (Gr), magnetic field inclination $(\theta)$ and oscillation frequency $(\omega)$. Complex variables are employed to solve the two-point boundary value problem. A steady state resonance of the velocity field is identified for $\omega>\frac{1}{2}\left(16 K^{4}-M^{4} \operatorname{Sin}^{4} \theta\right)^{1 / 2}$. Furthermore the solutions indicate that the condition $\omega>\frac{1}{2} \cos \omega T\left(16 K^{4}-M^{4} \operatorname{Sin}^{4} \theta\right)^{1 / 2}$ signifies an oscillatory turbulent dynamo mechanism. A critical Grashof number (Grcx) is also evaluated for which primary flow reversal arises at the upper channel plate. A similar criterion for Grashof number (Grcy) is established for the onset of secondary flow reversal at the upper plate. A detailed assessment of the influence of the control parameters on primary and secondary velocity evolution in the channel is also conducted. The model finds applications in MHD (Magneto Hydro Dynamic) energy generators and also magnetic materials processing systems.
\end{abstract}

Keywords: Magnetohydrodynamics (MHD), Free and forced thermal convection, Critical Grashof number, Rotation, Resonance, Complex variables solutions, Oblique magnetic field, MHD energy generators.

\section{INTRODUCTION}

MagnetoHydroDynamic (MHD) flows with and without heat transfer, arise in numerous areas of engineering and applied physics. A prominent area of focus is MHD energy generator flows which include disk systems (Yamasaki et al. (1988)), solar pond hydromagnetic generators (Kabakov and Yantovsky 1993) and magneto-thermo-acoustic generators (Vogin and Alemany 2006). Other applications arise in hypersonic ionized boundary layers (Macheret et al. 2004), particle deposition in electrically-conducting systems (Zueco et al. 2009) and liquid metal processing (Bég et al. 2009). In numerous hydromagnetic flows, rotation may also take place and the centrifugal forces can exert a significant effect on flow dynamics and heat transfer processes. Elco et al. (1962) studied analytically the characteristics of rotating flow in the radial vortex magneto-gas dynamic generator system. Yantovskiy and Tolmach (1963) investigated centrifugal force effects on rotating hydromagnetic generator configurations. Michiyoshi and Numano (1967) investigated the performance characteristics of the 
vortex MHD power generator using a partially ionized gas as a working fluid, showing that compressibility of the working fluid causes a much sharper decline of the radial velocity in the radial direction than in the case of an incompressible fluid at subsonic speeds. Further interesting studies of transient rotating hydromagnetic flow were reported by Katsurai (1972) and Oliver (1974). Lenzo et al (1978) studied experimentally the rotating-drum type separator in closed-cycle two-phase- MHD generator flow. power systems,. showing that a stable liquid film is formed on the drum and that the kinetic energy of the liquid layer is sufficient to be potentially useful in such energy generation systems. Thiagarajan and Rogthai (1977) analyzed the collisional plasma flow confined between co-axial cylindrical electrodes and rotating under crossed electric and magnetic fields, for the diffuse discharge regime, using 2-species MHD momentum equations, including Lorentz, pressure, centrifugal, Coriolis and collisional forces, with applications in MHD disk generators, isotope separation and plasma diagnostics. They found that retrograde motion (in part or whole of the annulus) arises over a range of magnetic fields for current flowing radially inward.

A number of mathematical and numerical studies of transient and rotating hydromagnetic flows have also appeared, employing a wide spectrum of analytical and computational methods. For example, oscillatory hydromagnetic flow in a continuous electrode Faraday generator was studied by Ibáñez et al. (2002), who also considered optimization aspects and derived generator efficiencies. Takhar et al. (1993) studied unsteady hydromagnetic flow of a dusty viscous liquid in a rotating channel with Hall currents and heat transfer, obtaining exact solutions and studying in detail the effect of Hartmann number and Strouhal number on the velocity evolution in the channel. Bég et al. (2010) used network simulation to study the radial, tangential and axial velocity fields and heat transfer in magneto-hydrodynamic flow from a spinning disk with thermal radiation and wall slip effects. Bég and Ghosh (2010) used complex variables to study the combined effects of thermal radiation and oscillating temperature on unsteady hydromagnetic boundary layer flow from a flat plate. Seth et al. (2010) used the Laplace transform technique to investigate transient rotating hydromagnetic Couette flow in a parallel porous plate channel, with flow induced due to the impulsive movement of the one of the plates of the channel. The plates of the channel are considered porous and the magnetic field. Asymptotic behavior of the solution was analyzed for small as well as large values of time and it was found that suction decelerates the primary as well as secondary flow where as injection and time have accelerating influence on the primary and secondary flows. Hayat et al. (2007) investigated Hall current magneto-hydrodynamics in rotating oscillating flows of a non-Newtonian fluid in a porous medium. Zueco and Bég (2010) used network simulation to study the transient magneto-elastohydrodynamic squeezing film flow between parallel rotating disks with magnetic induction effects. Ghosh and Bhattacharjee (2000) reported exact solutions for combined forced and free thermal convection hydromagnetic flow in rotating parallel plate channel with perfectly conducting walls, showing that shear stresses at the walls decrease with the increase in both the inverse Ekman number and Hartmann number squared and that the heat transfer rates at both walls decrease with the increase in the Grashof (free convection) number. Naroua et al. (2007) applied the finite element method to simulate unsteady magneto-hydrodynamic heat-generating free convection flow of a rotating partially-ionized gas past an infinite vertical porous plate with Hall and ion-slip current effects. They found that primary velocity profile decreases with a rise in the Hall parameter and the ionslip parameter, but is escalated with time for positive Grashof numbers (cooling of the plate by free convection currents) and decreases with time for negative Grashof numbers (heating of the plate by free convection currents). Secondary velocity profile was shown to be depressed with rising Hall parameter and ionslip parameter but elevated with increasing time and stronger rotation. Temperature profile was also shown to be boosted with a rise in the heat generating parameter and also with time. Ghosh et al. (2009) obtained analytical solutions for transient rotating magnetohydrodynamic free convection from a vertical surface embedded in a liquid-metal saturated Darcian porous medium. They considered both cases of heating of the plate by free convection currents (negative Grashof number) and cooling of the plate by free convection currents (positive Grashof number). Rawat et al. (2009) used a variational finite element scheme to numerically simulate the laminar, fully developed, transient MHD free convection heat and mass transfer of a conducting micropolar fluid between two vertical plates containing a non-Darcy porous medium. Strong deceleration of the flow with increasing magnetic field strength was established and also an accentuation of temperatures and concentration values of the dispersing agent indentified in the regime for strong magnetic fields. Ghosh et al. (2009) obtained both closed-form and asymptotic solutions for steady magnetohydrodynamic thermal convection in a spinning parallel plate channel system with perfectly conducting walls, Hall currents, magnetic induction and a static transverse uniform magnetic field. Joule (Ohmic) dissipation and viscous heating effects were also incorporated in the model. Boundary layers were identified to arise close to the channel walls for strong rotation of the channel. For slowly rotating systems, Hall current parameter was found to reduce primary mass flow rate. For constant values of the rotation parameter (inverse Ekman number), heat transfer rate at both plates was found to exhibit an oscillatory pattern with an increase in Hall current parameter. Bhargava et al. (2009) investigated the periodic reactive hydromagnetic free convection velocity, thermal and species diffusion boundary layers along a plate embedded in a porous medium with Soret and Dufour effects using an optimized variational finite element code. They showed that velocity is reduced with increasing magnetic parameter, whereas a rise increase in Eckert number (dissipation parameter) elevates temperature. Furthermore it was found that increasing chemical 
reaction parameter enhanced velocity, temperature and also concentration value, and that temperature was increased considerably with decreasing Soret number and simultaneous increasing Dufour number. Ghosh et al. (2009) derived exact solutions for the steady magnetohydrodynamic (MHD) rotating viscous convective flow of an optically-thin gas with thermal radiation and induced pressure gradient using the Laplace transform method. Both primary and secondary velocities were shown to increase with increasing rotation. An increase in Grashof number depressed both primary velocity and secondary velocity with significant backflow arising. Increasing magnetic field strength caused a deceleration in both the primary and secondary flow fields. Hayat et al. (2004) studied transient rotating MHD flow of a viscoelastic fluid analytically showing that a steady asymptotic hydromagnetic solution exists for blowing and resonance.

The above studies have all considered the case of a transverse magnetic field i.e. a magnetic field acting perpendicular to the principal flow direction. In many applications including astrophysics, MHD power generation and magnetic materials processing flow control, magnetic fields may act obliquely to the flow. Relatively few studies of inclined magnetic field effects on hydromagnetic transport phenomena have been presented. Several important studies have however been communicated in the past decade or so including Seth and Ghosh (1986) and Ghosh (1991). Ghosh $(1996,1997)$ considered the oblique magnetic field influence on MHD flow in a spinning channel in the presence of an oscillator. Ghosh (1999) also considered Hall effects on unsteady rotating channel hydromagnetics under an oblique magnetic field in the presence of an oscillator. Further analytical studies of such flows were described in Ghosh and Bhattacharjee (2000), Ghosh (2001) and Ghosh and Pop (2002, 2004). Ghosh and Pop (2006) investigated the hydromagnetic rotating plasma flow in the presence of an inclined magnetic field with the positive direction of the axis of rotation. The interplay of a hydromagnetic force and Coriolis force was shown to exert a strong influence of a dynamo mechanism with reference to the solar and terrestrial context when the Hall current is taken into account. This study also identified that the electrical discharge of the solar corona in the presence of a traveling magnetic field experiences an irregular fluctuation at the resonant level to produce a solar thermonuclear fusion reaction with regard to an excitation frequency. Ghosh et al. (2010) more recently obtained detailed network simulation numerical and also asymptotic and closed-form solutions for unsteady magneto-hydrodynamic flow in a rotating channel permeated by an inclined magnetic field with magnetic induction. This study also incorporated for the first time, the effect of Maxwell displacement current effects and simulated the case of ionized hydrogen in an MHD generator system. Bég et al. (2010) extended the Ghosh-Pop model (2002, 2004) to also consider porous media effects in rotating plasma flow with Hall currents and inclined magnetic field influence. Using a network simulation scheme they verified the analytical solutions of Ghosh and
Pop $(2002,2004)$ and also showed that complex flow characteristics arise owing to the imposition of a Darcian porous media drag force in the flow regime. Furthermore it was found that a sophisticated response to changing the orientation of the applied magnetic field is exhibited by the flow characteristics.

In the present study we shall consider the influence of an oblique magnetic field on the rotating transient viscous hydromagnetic flow and free and forced convection under a forced oscillation. This regime is sometimes referred to as the "magnetic mirror regime" in astrophysical fluid dynamics. The present model has applications however both in astrophysical flows and also in hybrid MHD energy generator exploiting variable orientation magnetic fields. Such a study has to the authors' knowledge, thus far, not appeared in the literature and is an important extension to hydromagnetic thermal sciences research.

\section{Mathematical ModeL}

Consider the transient, magneto-hydrodynamic free and forced convection flow of a viscous, incompressible, electrically-conducting Newtonian fluid between parallel plates, located a distance $2 \mathrm{~L}$ apart, along the $\mathrm{z}$ - axis, under the action of a constant pressure gradient, in an $(x, y, z)$ coordinate system. We choose a Cartesian system such that the $\mathrm{z}-$ axis is perpendicular to the plates $\mathrm{z}= \pm \mathrm{L}$.

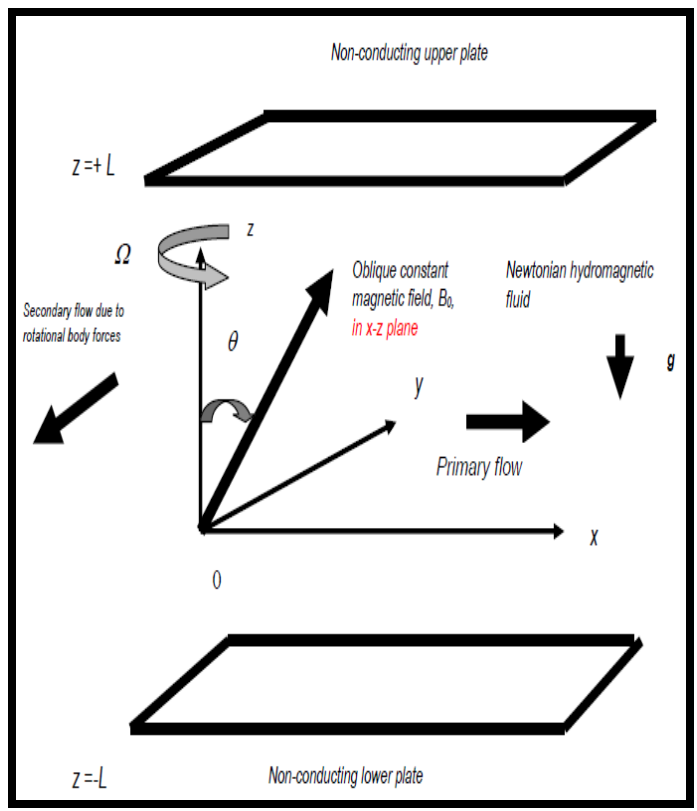

Fig. 1. Physical model and coordinate system for MHD rotating channel flow

The $\mathrm{x}-$ axis is in the direction of the pressure gradient. The channel rotates with uniform angular velocity, $\Omega$, about the $\mathrm{z}$-axis (rotation axis) transverse to the plane of the flow ( $\mathrm{x}-\mathrm{y}$ plane), under a static (non-oscillating), uniform magnetic field, $B_{0}$ 
The inclined magnetic field, $B_{0}$, is orientated at angle $\theta$ to the positive $\mathrm{z}$-axis, in the $\mathrm{x}-\mathrm{z}$ plane. Therefore the angle will sweep in a clockwise fashion, a circle only in the $\mathrm{x}-\mathrm{z}$ plane: $\theta$ will increase from 0 (when $B_{0}$ is directed along the positive vertical $\mathrm{z}$-axis direction) to 90 degrees i.e $\pi / 2$, (when $B_{0}$ is directed along the positive $\mathrm{x}$-axis direction), to $\pi$ (when $B_{0}$ is directed along the negative $\mathrm{z}$-axis direction), to $3 \pi / 2$ (when $B_{0}$ is directed along the negative $\mathrm{x}$-axis direction) and return to 0 degrees orientation (when $B_{0}$ is again directed along the positive vertical $\mathrm{z}$-axis direction). The regime is illustrated in Fig. 1 below. Both the fluid and the channel rotate in unison as a rigid body with the same constant angular velocity of rotation. Since the flow is influenced by a forced oscillation a time-varying current flow of sinusoidal nature becomes relevant to the case of a displacement current with reference to the inclined magnetic field. The channel plates are both electrically non-conducting. Magnetic Reynolds number is insufficient to invoke magnetic induction effects, and furthermore ion slip, Hall current and Alfven waves are neglected in the analysis. The plates are infinite along the $\mathrm{x}$ and $\mathrm{y}$ directions, and therefore all physical quantities with the exception of pressure will be functions of the independent spatial and temporal variables, $\mathrm{z}$ and $\mathrm{t}$ (time) only Following Hughes and Young (1966), we take the following vectorial field definitions, which are customary in engineering magneto-fluid dynamics

$\boldsymbol{q}=\left(u^{\prime}, v^{\prime}, 0\right)$

$\boldsymbol{B}=\left(B^{\prime}{ }_{x}+B_{0} \sin \theta, B^{\prime}{ }_{y}, B_{0} \cos \theta\right)$

$\boldsymbol{E}=\left(E_{x}, E_{y}, E_{z}\right)$

$\boldsymbol{J}=\left(J_{x}, J_{y}, J_{z}\right)$

where q, B, E, J are, respectively, the velocity vector $(\mathrm{m} / \mathrm{s})$, the applied magnetic field vector (Tesla i.e. Webers $/ \mathrm{m} 2$ or Volt-seconds $/ \mathrm{m} 2$ ), the electric field vector (Volts $/ \mathrm{m}$ or $\mathrm{N} /$ Coulomb) and the current density vector (Coulombs/m2 or N/Volt-metre). u' is the $\mathrm{x}$ - component of velocity, $\mathrm{v}^{\prime}$ is the $\mathrm{y}$ - component of velocity, B'x is the $\mathrm{x}$ - component of applied magnetic field, B'y is the $\mathrm{y}-$ component of applied magnetic field, $B_{0}$ is the magnetic flux density and $\theta$ is the angle of inclination of the applied magnetic field with the positive direction of the axis of rotation ( $\mathrm{z}-$ axis). Ex, Ey and $\mathrm{Ez}$ are respectively, the components of electric field in the $x, y$ and $z$ directions. Jx, Jy and $\mathrm{Jz}$ are respectively, the $\mathrm{x}, \mathrm{y}, \mathrm{z}$ components of current densities.

The equations of motion (Navier-Stokes MHD momentum equations) under the Boussinesq approximation,for the transient magnetohydrodynamic rotating channel flow under oblique magnetic field, can then be shown to assume the form:

$$
\begin{aligned}
& \frac{\partial \boldsymbol{q}}{\partial t}+(\boldsymbol{q} \cdot \nabla) \boldsymbol{q}+2 \Omega \boldsymbol{k} \times \boldsymbol{q}=-\frac{1}{\rho} \nabla p+\nu \nabla^{2} \boldsymbol{q}+ \\
& \frac{1}{\rho} \boldsymbol{J} \times \boldsymbol{B}+\boldsymbol{g}\left[1-\beta^{\prime}\left(T-T_{0}\right)\right] \boldsymbol{k}
\end{aligned}
$$

The equation of continuity (mass conservation) is

$$
\nabla \cdot \boldsymbol{q}=0
$$

Maxwell's electromagnetic field equations, following Hughes and Young (1966), neglecting the Maxwellian displacement currents, may be stated:

$$
\begin{aligned}
& \nabla X B=\mu_{e} J \quad \text { (Ampére's law) } \\
& \nabla X E=-\frac{\partial \boldsymbol{B}}{\partial t} \text { (Faraday's law) } \\
& \nabla \cdot B=0 \quad \text { (solenoidal relation) } \\
& \nabla . E=\frac{\rho}{\varepsilon_{0}} \quad \text { (Gauss' law) }
\end{aligned}
$$

Equations (4a) and (4b) effectively describe how electric charges produces electromagnetic fields. Ohm's law for a moving conductor, neglecting Hall current takes the form:

$$
\boldsymbol{J}=\sigma[\boldsymbol{E}+\boldsymbol{q} \times \mathbf{B}]
$$

Equation (5) effectively shows how the fields affect the charges. The following notation applies: $\Omega$ is angular velocity (radians/s), $\varepsilon_{0}$ is permittivity of free space which is also known as the electric constant (Farads per meter), $t$ is time (s), $g$ is gravity $\left(\mathrm{m} / \mathrm{s}^{2}\right), \rho$ is the fluid density $\left(\mathrm{kg} / \mathrm{m}^{3}\right), v$ is kinematic viscosity $\left(\mathrm{m}^{2} / \mathrm{s}\right), \mu_{e}$ is the magnetic permeability (Henrys per meter, or N/Amp $\left.{ }^{2}\right) p$ is pressure $(\mathrm{Pa}), \sigma$ is the fluid electrical conductivity (Siemens/metre), $\beta$ is the coefficient of volume expansion, $T$ is fluid temperature (Kelvins), $T_{0}$ is the temperature in the reference state (Kelvins), $B_{0}$ is the applied magnetic flux density (the magnetic induction, also called the magnetic field density or magnetic flux density with units, Tesla) and $\boldsymbol{k}$ is the unit vector directed along the $z$-axis (rotation axis). Since there is no electrical field applied in the current regime under consideration (Fig. 1), the polarization voltage is neglected. Therefore it follows that $\boldsymbol{E}=0$, as indicated by Meyer (1958). Under these simplifications, the governing conservations equations, in component form, may be stated as follows:

\section{$x$-momentum}

$\frac{\partial u^{\prime}}{\partial t}-2 \Omega v^{\prime}=-\frac{1}{\rho} \frac{\partial p}{\partial x}+v \frac{\partial^{2} u^{\prime}}{\partial z^{2}}-\frac{\sigma B_{0}^{2}}{\rho} u^{\prime} \cos ^{2} \theta$,

y-momentum

$\frac{\partial v^{\prime}}{\partial t}+2 \Omega u^{\prime}=v \frac{\partial^{2} v^{\prime}}{\partial z^{2}}-\frac{\sigma B_{0}^{2}}{\rho} v^{\prime}$

\section{z-momentum}

$0=-\frac{1}{\rho} \frac{\partial p}{\partial z}+g\left\{1-\beta^{\prime}\left(T-T_{0}\right)\right\}+\frac{\sigma B_{0}{ }^{2}}{\rho} u^{\prime} \sin \theta \cos \theta$.

The appropriate boundary conditions (no-slip) at the plates are prescribed as:

$u^{\prime}=v^{\prime}=0 \quad$ at $\quad z= \pm L$

$u^{\prime}, v^{\prime}$ are velocity components in the $x$ and $y$ directions, respectively, $\theta$ is magnetic field orientation (degrees) and all other parameters have 
been hitherto defined. The final terms in Eqs. (6) and (8) designate the components of the Lorentzian hydromagnetic retarding force. Assuming uniform axial temperature variation along the plates of the channel, the temperature of the fluid may be written, following Mazumder (1977) and Mazumder et al. (1976) as:

$$
\left(T-T_{0}\right)=N x+\varphi(z)
$$

where $N$ is constant (uniform wall temperature gradient parameter) and the other terms have been defined earlier. The equation of state is:

$\rho=\rho_{0}\left[1-\beta^{\prime}\left(T-T_{0}\right)\right]$

Such a model while less complex than the conventional nonlinear convective heat transfer equation model, does nevertheless simulate one of the objectives of the present study - the net effect of buoyancy on momentum development in the channel. Albeit an approximation, this model has justifications and has been successfully employed in recent years. Mazumder (1977) has successfully implemented this model yielding extremely accurate results for astronautical heat transfer problems. Mazumder et al. (1976) also utilized this model to excellent effect in their simulation of Hall current effects on composite natural and forced convection heat transfer in magnetohydrodynamic channel flow. $\Phi(z)$ is dependent on the independent variable, $z$. Integration of Eq. (8) and subsequent differentiation with respect to $\mathrm{x}$ leads to $d \Phi(z) / d x$ being zero. Now from Eq. (10), we have $T-T_{0}=N x+\Phi(z)$, where $N$ is the uniform temperature gradient of the wall (i.e. wall temperature gradient parameter). $\Phi(z)$ is considered as the amount of heat transferred across the surface (wall) i.e. the wall heat flux. This methodology provides a reasonable engineering approximation for the present problem. Using Eq. (11) and integrating Eq. (8) effectively leads to

$$
\begin{aligned}
p= & -\rho_{0} g \int\left[1-\beta^{\prime}\left(T-T_{0}\right)\right] d z \\
& +\frac{\sigma B_{0}{ }^{2}}{2} z u^{\prime} \sin 2 \theta+F(x)
\end{aligned}
$$

Combining Eqs (10) and (12) we have

$$
\frac{\partial p}{\partial x}=-\rho g \beta^{\prime} N z+\frac{d}{d x} F(x)
$$

where $F(x)$ is an arbitrary function. Finally using Eq. (13), then Eq (6) yields:

$$
\begin{array}{r}
\frac{\partial u^{\prime}}{\partial t}-2 \Omega v^{\prime}=g \beta^{\prime} N z-\frac{1}{\rho} \frac{d}{d x}\{F(x)\} \\
+v \frac{\partial^{2} u^{\prime}}{\partial z^{2}}-\frac{\sigma B_{0}^{2}}{\rho} u^{\prime} \cos ^{2} \theta,
\end{array}
$$

Equations (14), (7) and (8) under boundary conditions Eq. (9) constitute a robust two-point boundary value problem. This model can be solved in primitive variable form either numerically or with analytical tools. However to yield a more generalized understanding of the flow phenomena, it is pertinent and beneficial to introduce normalized variables. This also facilitates the evaluation of analytical solutions.

\section{Model Transformation}

Proceeding with the analysis, we now introduce the following non-dimensional variables:

$$
\begin{aligned}
& u=\left(\frac{L}{v p_{x}}\right) u^{\prime} \\
& v=\left(\frac{L}{v p_{x}}\right) v^{\prime} \\
& t=\frac{L^{2} T}{v^{\prime}} \\
& P_{x}=-\frac{L^{3}}{\rho v^{2}} \frac{d F}{d x} \\
& G r=\frac{g \beta N L^{4}}{v^{2} p_{x}} \\
& M^{2}=B_{0}{ }^{2} L^{2}\left(\frac{\sigma}{\rho v}\right)^{1 / 2} \\
& \eta=\frac{z}{L} \\
& K^{2}=\frac{\Omega L^{2}}{v}
\end{aligned}
$$

where $\eta$ is dimensionless $\mathrm{z}$-coordinate, $\mathrm{u}$ and $\mathrm{v}$ are non-dimensional primary and secondary velocities, $\mathrm{T}$ is dimensionless time, $P x$ is non-dimensional pressure gradient in the x'-direction, $\mathrm{Gr}$ is Grashof number, $M^{2}$ is the Hartmann hydromagnetic number, and $K^{2}$ is the rotation parameter i.e. reciprocal of the Ekman number. Introducing the transformations' Eqs. (15a) to (15h) into Esq. (14), (7) and (8) and boundary conditions Eq. (9), results in the following dimensionless equations and boundary conditions, with the elimination of the z-momentum equation:

\section{Primary Momentum Equation}

$\frac{\partial^{2} u}{\partial \eta^{2}}-\frac{\partial u}{\partial T}-M^{2} u \cos ^{2} \theta=-1-G r \eta-2 K^{2} v$,

\section{Secondary Momentum Equation}

$$
\frac{\partial^{2} v}{\partial \eta^{2}}-\frac{\partial v}{\partial T}-M^{2} v=2 K^{2} u \text {. }
$$

\section{Transformed Boundary Conditions}

$$
u=v=0 \quad \text { at } \quad \eta= \pm 1
$$

\section{Analytical Solution}

Since the hydromagnetic flow is influenced by a forced oscillation, the following expressions are implemented for the velocity fields:

$u(\eta, T)=u_{0}(\eta) \cos \omega T$ 
$v(\eta, T)=v_{0}(\eta) \cos \omega T$

Utilizing expressions Eqs. (19a) and (19b), the velocity distributions obtained for the primary (main) and secondary (cross) flows are, based on the method of complex variables.

This technique is one of the most powerful methods available in applied mathematics and fluid mechanics. It is concerned with complex functions which are differentiable in a given domain. In MHD we employ analytical functions with complex variables. A function $\mathrm{f}(\mathrm{z})$ is defined as analytic (Bég et al. 2011) in a domain $\mathrm{D}$ if $\mathrm{f}(\mathrm{z})$ is defined and differentiable at all points of $D$. the function $f(z)$ is analytic at a point $\mathrm{z}=\mathrm{z} 0$ in $\mathrm{D}$, provided $\mathrm{f}(\mathrm{z})$ is analytic in a neighborhood. The necessary condition therefore for implementation of complex variables in e.g. magneto-hydrodynamic flows, is that the function $\mathrm{f}(\mathrm{z})$ must be differentiable not only at a point $\mathrm{z} 0$, but throughout a specified neighborhood of that point. Such functions are also known as holomorphic in the domain D. Using this approach, the complex solutions can be shown to take the form:

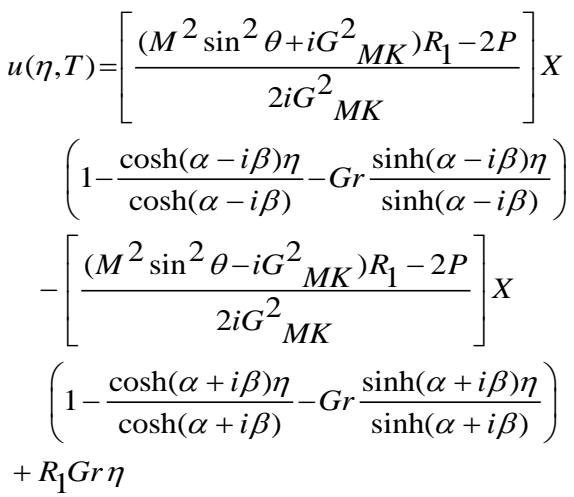

$v(\eta, T)=\left[\frac{2 P\left(M^{2} \sin ^{2} \theta-i G_{M K}^{2}\right)-\left(M^{4} \sin ^{4} \theta+G_{M K}^{4}\right) R_{1}}{8 i K^{2} G^{2}{ }_{M K}}\right]$

$\left(1-\frac{\cosh (\alpha-i \beta) \eta}{\cosh (\alpha-i \beta)}-G r \frac{\sinh (\alpha-i \beta) \eta}{\sinh (\alpha-i \beta)}\right)$

$-\left[\frac{2 P\left(M^{2} \sin ^{2} \theta+i G^{2}{ }_{M K}\right)-\left(M^{4} \sin ^{4} \theta+G_{M K}^{4}\right) R_{1}}{8 i K^{2} G^{2}{ }_{M K}}\right] X$

$\left(1-\frac{\cosh (\alpha+i \beta) \eta}{\cosh (\alpha+i \beta)}-G r \frac{\sinh (\alpha+i \beta) \eta}{\sinh (\alpha+i \beta)}\right)-\frac{P}{2 K^{2}} G r \eta$

where

$R_{1}=\frac{M^{2}}{\left(\alpha^{2}+\beta^{2}\right)^{2}}$

$P=1-\frac{M^{2}\left[M^{2} \cos ^{2} \theta-\omega \tan \omega T\right]}{\left(\alpha^{2}+\beta^{2}\right)^{2}}$

$$
\begin{aligned}
& H=\left(1+\cos ^{2} \theta\right) M^{2}-2 \omega \tan \omega T \\
& \alpha, \beta=\frac{1}{2}\left[\left(H^{2}+G^{4}{ }_{M K}\right)^{1 / 2} \pm H\right]^{1 / 2} \\
& G_{M K}^{2}=\left(16 K^{4}-M^{4} \sin ^{4} \theta-4 \omega^{2} \tan ^{2} \omega T-4 \omega^{2}\right)^{1 / 2}
\end{aligned}
$$

\section{Special Cases}

From the general solutions obtained in section 4 , we may derive briefly some special cases, for physically pertinent regimes.

\section{Case I: Oscillating Forced convection with an} Oblique Magnetic Field

In the absence of buoyancy forces $\mathrm{Gr} \rightarrow 0$ ) the solutions Eqs. (20) and (21) reduce to the case for pure forced magnetohydrodynamic convection:

$$
\begin{aligned}
& u(\eta, T)= \frac{\left(M^{2} \sin ^{2} \theta+i G_{M K}^{2}\right) R_{1}-2 P}{2 i G^{2} M K} X \\
&\left\{1-\frac{\cosh (\alpha-i \beta) \eta}{\cosh (\alpha-i \beta)}\right\}- \\
& \frac{\left(M^{2} \sin ^{2} \theta-i G^{2}{ }_{M K}\right) R_{1}-2 P}{2 i G^{2}{ }_{M K}} X \\
&\left\{1-\frac{\cosh (\alpha+i \beta)}{\cosh (\alpha+i \beta)}\right\} \\
& v(\eta, T)= \frac{2 P\left(M^{2} \sin ^{2} \theta-i G^{2}{ }_{M K}\right)-D R_{1}}{8 i K^{2} G^{2}{ }_{M K}} X \\
&\left\{1-\frac{\cosh (\alpha-i \beta) \eta}{\cosh (\alpha-i \beta)}\right\}- \\
& \frac{2 P\left(M^{2} \sin ^{2} \theta+i G^{2}{ }_{M K}\right)-D R_{1}}{8 i K^{2} G^{2}{ }_{M K}} X \\
&\left\{1-\frac{\cosh (\alpha+i \beta) \eta}{\cosh (\alpha+i \beta)}\right\}
\end{aligned}
$$

With

$$
D=M^{4} \sin ^{4} \theta+G^{4} M K
$$

The expressions Eqs. (22a) to (22d) will remain unchanged. This regime is of considerable interest in MHD generators, as elaborated in Vogin and Alemany (2007).

\section{Case II: Oscillating Free Convection with a Transverse Magnetic Field}

With $\theta \rightarrow 0$, the applied magnetic field, $B_{0}$ will become orientated along the $z$-axis i.e. at exactly 90 degrees ( $\pi / 2$ radians) to the $x-y$ plane of flow. Clearly $\sin 0 \rightarrow 0$ and $\cos 0 \rightarrow 1$. Effectively Lorentzian hydromagnetic drag will be retained in both the primary flow velocity, and secondary flow velocity, via the $P, \alpha$ and $\beta$ expressions which will still retain $M^{2}$ terms. Magnetic field, $M^{2}$ however will disappear from the expression, $G_{M K}$. The corresponding solutions for the primary and secondary flow fields, respectively, will then reduce from expressions (20) and (21) to: 


$$
\begin{aligned}
u(\eta, T) & =\left[\frac{\left(i G^{2} M K\right) R_{1}-2 P}{2 i G^{2} M K}\right] X \\
& \left(1-\frac{\cosh (\alpha-i \beta) \eta}{\cosh (\alpha-i \beta)}-G r \frac{\sinh (\alpha-i \beta) \eta}{\sinh (\alpha-i \beta)}\right) \\
& -\left[\frac{\left(-i G^{2}{ }_{M K}\right) R_{1}-2 P}{2 i G^{2} M K}\right] X \\
& \left(1-\frac{\cosh (\alpha+i \beta) \eta}{\cosh (\alpha+i \beta)}-G r \frac{\sinh (\alpha+i \beta) \eta}{\sinh (\alpha+i \beta)}\right)+R_{1} G r \eta
\end{aligned}
$$

$$
\begin{gathered}
v(\eta, T)=\frac{2 P\left(-i G^{2}{ }_{M K}\right)-R_{1} G^{4}{ }_{M K}}{8 i K^{2} G^{2} M K} X \\
\left(1-\frac{\cosh (\alpha-i \beta) \eta}{\cosh (\alpha-i \beta)}-G r \frac{\sinh (\alpha-i \beta) \eta}{\sinh (\alpha-i \beta)}\right) \\
-\frac{2 P\left(i G^{2}{ }_{M K}\right)-R_{1} G^{4} M K}{8 i K^{2} G^{2} M K} X \\
\left(1-\frac{\cosh (\alpha+i \beta) \eta}{\cosh (\alpha+i \beta)}-G r \frac{\sinh (\alpha+i \beta) \eta}{\sinh (\alpha+i \beta)}\right)-\frac{P}{2 K^{2}} G r \eta
\end{gathered}
$$

In this case, the expressions Eqs. (22b) to (22e) will be affected and reduce to:

$$
\begin{aligned}
& P=1-\frac{M^{2}\left[M^{2}-\omega \tan \omega T\right]}{\left(\alpha^{2}+\beta^{2}\right)^{2}} \\
& \alpha, \beta=\frac{1}{2}\left[\left(C^{2}+G^{4}{ }_{M K}\right)^{1 / 2} \pm C\right]^{1 / 2} \\
& G_{M K}^{2}=\left(16 K^{4}-4 \omega^{2} \tan ^{2} \omega T-4 \omega^{2}\right)^{1 / 2} \\
& C=2 M^{2}-2 \omega \tan \omega T
\end{aligned}
$$

Of course the expression Eq. (22a) i.e. $R_{l}$ is affected, since $\alpha, \beta$ are affected. This case provides an excellent benchmark for the vast majority of studies in oscillatory hydromagnetics, which consider only a transverse magnetic field acting on the flow.

\section{Case III: Oscillatory "Magnetic Mirror" buoyant Convection with Oblique Magnetic Field}

With the angular frequency of oscillation, $\omega T \rightarrow \pi / 2$, a resonant response arises corresponding to the condition, $\omega>\frac{1}{2} \cos \omega T\left(16 K^{4}-M^{4} \operatorname{Sin}^{4} \theta\right)^{1 / 2}$. In an astrophysical context this implies that laser radiation corresponds to a driving frequency in the presence of a magnetic mirror, when $\omega>0$ (driving frequency). A resonant response will arise; laser radiation will be of sufficient intensity in the presence of a magnetic mirror to generate reflection in the form of ultraviolet radiation and binary X-rays as the magnetic field increase in abruptly. The condition, $\omega>\frac{1}{2} \cos \omega T\left(16 K^{4}-M^{4} \operatorname{Sin}^{4} \theta\right)^{1 / 2} \quad$ defines an oscillatory turbulent dynamo mechanism in a solar hydromagnetic regime if the strength of a magnetic field will be an appropriate level. A charged oscillation can take place with reference to a driving force to exhibit resonant fluorescence in the presence of an ( driving frequency ) excitation frequency, $\omega$ $>0$.

In the context of engineering energy systems, this case has particularly significant applications in the thermo-acoustic MHD generator, which exploits an imposed harmonically-oscillating thermo-acoustically generated pressure gradient. Kolesnikov and Khait (1975) earlier studied forced convection oscillatory flow in MHD generators, obtaining a solution in series form with allowance for terms expressing the square of the amplitude of (dimensionless) velocity fluctuations. However unlike the present study, the investigations by Vogin and Alemany (2007) and Kolesnikov and Khait (1975) have not considered rotational effects. In plasma fusion energy systems, also the magnetic mirror design is very promising. Discovered in astrophysical plasma flows, a magnetic mirror, comprises a magnetic field configuration where the field strength changes when moving along a field line. The mirror effect results in a tendency for charged particles to bounce back from the high field region. Charged particles with a velocity component perpendicular to the field will gyrate around a field line in a generally circular or helical orbit and thus sample some of the field lines that are converging to create the field gradient. The radial component of these field lines, coupled with the azimuthal motion of the particle, will result in a force parallel to the field and directed toward the region of smaller field strength. Over three decades ago, engineers began to study systems using magnetic mirror confinement for producing fusion energy. The lingering difficulty with such systems was the complexity of sustaining the necessary non-Maxwellian velocity distribution, since collisions scattered the charged particles so that the pitch angle was too small for containment. In addition, velocity space instabilities increased the escape of the plasma. However recently Sokoloff (2007) has re-examined such systems (studied earlier by Frank et al. 1977), in particular magnetic mirrors used in next generation Tokamak magnetic fusion energy devices where the toroidal magnetic field is stronger on the inboard side than on the outboard side. The resulting effects are known as neoclassical. As we have mentioned earlier, magnetic mirrors also arise in astrophysical regimes e.g. electrons and ions in the magnetosphere (Moir, 1977 and Rice and Schaerer, 1986) for example, will bounce back and forth between the stronger fields at the poles. The Coriolis force acting on rotating vortices in a stratified media results in an excess of right-hand vortices in one hemisphere and left-hand vortices in the other. This asymmetry gives a component of the mean electromotive force parallel to the mean magnetic field (the electromotive force and the electric current are perpendicular to the magnetic field in mirror-symmetric media). This is the famous alpha-effect which plays a key role in magnetic field 
self-excitation (the so-called mean-field dynamo) and leads to the solar cycle and other phenomena in astrophysical plasma. At the temperatures required for engineering fusion devices, the fuel is in the form of a plasma with very good electrical conductivity. This facilitates the possibility to confine the fuel and the energy with magnetic fields, an idea known as magnetic confinement (Ferreira et al. 2004). The Lorentz force works only perpendicular to the magnetic field, so that the first problem is how to prevent the plasma from leaking out the ends of the field lines. Employing a magnetic mirror, when particles following a field line encounter a region of higher field strength, then some of the particles will be stopped and reflected. Advantages of a magnetic mirror power plant would therefore be simplified construction and maintenance due to a linear topology and the potential to apply direct conversion in a natural way.

\section{Case IV: Steady State Resonant Free Convection with an Oblique Magnetic Field}

With the angular frequency of oscillation, $\omega T \rightarrow 0$, oscillation is eliminated in the regime. The regime is then "steady state". The velocity distributions in Eqs. (20) and (21), can be expounded in two ways. The excitation frequency may be either $\omega<\frac{1}{2}\left(16 K^{4}-M^{4} \operatorname{Sin}^{4} \theta\right)^{1 / 2}$ or $\omega>\frac{1}{2}\left(16 K^{4}-M^{4} \operatorname{Sin}^{4} \theta\right)^{1 / 2}$. The former condition corresponds to a low frequency of oscillation in response to a solar dynamo mechanism, wherein the Lorentzian (magnetohydrodynamic) and Coriolis (rotational) forces are of the same order of magnitude. The latter condition implies a resonant response of turbulent characteristics, and the flow regime is destabilized by a magnetic field. This latter condition therefore leads to a steady state resonance on the velocity field.

\section{SHEAR STRESS Distribution}

Since we are primarily concerned with terrestrial (engineering) applications of the flow configuration, it is pertinent to define several key quantities from an engineering design point, of relevance to MHD energy generators. The primary and secondary shear stresses at the upper and lower plates can be derived by taking the first gradients of the respective velocities at the lower $(\eta=-1)$ and upper $(\eta=+1)$ plates.

Shear stresses at the upper and lower plates $\eta= \pm 1$ can be easily obtained from Eqs. (20) and (21) with reference to $\frac{d u}{d \eta} \mid \eta= \pm 1$ and $\frac{d v}{d \eta} \mid \eta= \pm 1$.

Inspection of $\left.\frac{d u}{d \eta}\right|_{\eta= \pm 1}$ and $\left.\frac{d v}{d \eta}\right|_{\eta= \pm 1}$ reveal that the primary (main flow) and secondary (cross flow) shear stress components vanish neither on the upper plate or the lower plate. Both shear stresses are functions of magnetic field $\left(M^{2}\right)$, rotational parameter $\left(K^{2}\right)$, phase angle $(\omega T)$ and of course magnetic field orientation $(\theta)$. Closer inspection of the shear stress expressions indicates that in the forced convection case $(G r \rightarrow 0)$, flow reversal i.e. backflow, will not arise. Further analysis however indicates that the primary shear stress at the lower plate i.e. $\left.\frac{d u}{d \eta}\right|_{\eta=-1}$ will vanish at a critical value of the Grashof number. This criterion is defined as:

$$
G r_{c x}=-\frac{\cosh 2 \alpha-\cos 2 \beta}{\cosh 2 \alpha+\cos 2 \beta} \frac{F_{5} G_{M K}^{2}-F_{4}}{F_{3} G_{M K}^{2}+F_{2}}
$$

where the functions $F_{1}, F_{2}, F_{3}, F_{4}$ and $F_{5}$ are defined respectively as:

$$
\begin{aligned}
& F_{1}=M^{4} \sin ^{2} \theta-8 K^{4} \\
& +2 M^{2} \omega \cos ^{2} \theta \tan \omega T+2 \omega^{2} \\
& F_{2}=F_{1}(\beta \sinh 2 \alpha-\alpha \sin 2 \beta) \\
& F_{3}=M^{2}(\cosh 2 \alpha-\cos 2 \beta- \\
& \alpha \sinh 2 \alpha-\beta \sin 2 \beta) \\
& F_{4}=F_{1}(\beta \sinh 2 \alpha+\alpha \sin 2 \beta) \\
& F_{5}=M^{2}(\alpha \sinh 2 \alpha-\beta \sin 2 \beta)
\end{aligned}
$$

Since $\cosh 2 \alpha>\cos 2 \beta$ for all $K^{2}, \omega T$ and $\theta$, the numerator in Eq. (28) will always be positive.

The secondary (cross flow) shear stress at the lower plate, i.e. $\left.\frac{d v}{d \eta}\right|_{\eta=-1}$ will also vanish at a critical value of the Grashof number. This criterion is defined as:

$G r_{c y}=-\frac{\cosh 2 \alpha-\cos 2 \beta}{\cosh 2 \alpha+\cos 2 \beta} \frac{2 H_{6} G_{M K}^{2}-H_{5}}{H_{3}+2 H_{4} G_{M K}^{2}}$

where the functions $H_{1}, H_{2}, H_{3}, H_{4}, H_{5}$ and $H_{6}$ are defined respectively as

$$
\begin{aligned}
& H_{1}=4 K^{4}-M^{2} \omega \cos ^{2} \theta \tan \omega T-\omega^{2} \\
& H_{2}=M^{2}\left[M^{4} \sin ^{4} \theta+G_{M K}^{4}\right] \\
& -2 H_{1} M^{2} \sin ^{2} \theta \\
& H_{3}=H_{2}(\beta \sinh 2 \alpha-\alpha \sin 2 \beta)
\end{aligned}
$$


$H_{4}=H_{1}(\cosh 2 \alpha-\cos 2 \beta-\alpha \sinh 2 \alpha$

$-\beta \sin 2 \beta)$

$H_{5}=H_{2}(\alpha \sin 2 \beta+\beta \sinh 2 \alpha)$

$H_{6}=H_{1}(\alpha \sinh 2 \alpha-\beta \sin 2 \beta)$

Proceeding as with the lower plate, we find that at the upper plate, primary (main) flow reversal is initiated when:

$G r_{c x}=\frac{\cosh 2 \alpha-\cos 2 \beta}{\cosh 2 \alpha+\cos 2 \beta} \frac{F_{5} G_{M K}^{2}-F_{4}}{F_{3} G_{M K}^{2}+F_{2}}$

Similarly secondary (cross) flow reversal arises at the upper plate when:

$$
G r_{c y}=\frac{\cosh 2 \alpha-\cos 2 \beta}{\cosh 2 \alpha+\cos 2 \beta} \frac{2 H_{6} G_{M K}^{2}-H_{5}}{H_{3}+2 H_{4} G_{M K}^{2}}
$$

The critical Grashof numbers given by expressions Eqs. (30) and (31) will therefore be numerically equal to those yielded by expressions Eqs. (26) and (28), for the primary flow, since the expressions emerge as being the same.

\section{RESUlt AND DiscuSSION}

We have obtained solutions for the fourth order ordinary coupled partial differential equation system defined by Eqs. (16), (17) subject to a forced oscillation given by the Eqs. (19 a) and (19b) based on the boundary conditions Eq. (18). This yields the exact solutions given in Eqs. (20) and (21). The evolution of dimensionless primary and secondary velocity distributions given by the Eqs. (20) and (21) with the inclusion of Eq. (22) due to primary and secondary flows have been depicted graphically to show the influence of the various controlling parameters viz., $M^{2}, K^{2}, \theta, G r$ and $\omega T$. A parametric study has also been undertaken for theresponse of the critical Grashof numbers for the primary and secondary flows $\left(G r_{c x}, G r_{c y}\right)$ defined in Eqs. (30) and (31) respectively, to $\theta$ (magnetic field inclination), $M^{2}$ (Hartmann number squared), $K^{2}$ (inverse Ekman number) and $\omega T$ (phase angle). These are depicted in Tables 1 to 6 . Additionally numerical evaluations of the dimensionless primary $(u)$ and secondary $(v)$ velocities have been conducted for both steady state $(\omega T=0)$ and transient $(\omega T>0)$ cases, for the effects of $\theta$ (inclination), $M^{2}$ (Hartmann number squared), $K^{2}$ (inverse Ekman number) and $\mathrm{Gr}$ (Grashof number). These are plotted in figures in Fig. 2 to Fig. 10. Default values for the control parameters in these graphs are taken as $K^{2}=4 ; \omega T=0$ (steady state) or $\omega T=\pi / 4$ (transient i.e. oscillatory flow) and $\omega$ (oscillation frequency) $=0.4$ (so that $T=1), M^{2}=10$ (strong magnetic field) and $\theta=\pi / 4$, unless otherwise stated

Table 1 Critical Grashof number for primary flow $\left(G r_{c x}\right)$ for $K^{2}=5, \omega T=\pi / 4, \omega=0.4$, with $M^{2}$ and $\theta$ variation.

\begin{tabular}{|c|c|c|c|c|}
\hline$M^{2} \rightarrow$ & 2.0 & 5.0 & 8.0 & 10.0 \\
\hline$\theta \downarrow$ & $\mathbf{G r}_{\mathbf{c x}}$ & $\mathbf{G r}_{\mathbf{c x}}$ & $\mathbf{G r}_{\mathbf{c x}}$ & $\mathbf{G r}_{\mathrm{cx}}$ \\
\hline 0 & 1.08222 & 1.20181 & 1.25510 & 1.26797 \\
\hline$\pi / 6$ & 1.07974 & 1.20510 & 1.27004 & 1.29005 \\
\hline$\pi / 4$ & 1.07708 & 1.20844 & 1.28810 & 1.318607 \\
\hline$\pi / 3$ & 1.07423 & 1.21182 & 1.310601 & 1.35745 \\
\hline$\pi / 2$ & 1.07117 & 1.21520 & 1.33969 & 1.41411 \\
\hline
\end{tabular}

Table 2 Critical Grashof number for secondary flow $\left(G r_{c v}\right)$ for $K^{2}=5, \omega T=\pi / 4, \omega=0.4$, with $M^{2}$ and $\theta$ variation.

\begin{tabular}{|c|c|c|c|c|}
\hline$M^{2} \rightarrow$ & 2.0 & 5.0 & 8.0 & 10.0 \\
\hline$\theta \downarrow$ & $\mathbf{G r}_{\mathbf{c y}}$ & $\mathbf{G r}_{\mathbf{c y}}$ & $\mathbf{G r}_{\mathbf{c y}}$ & $\mathbf{G r}_{\mathbf{c y}}$ \\
\hline 0 & 2.15679 & 2.22039 & 2.16832 & 2.10984 \\
\hline$\pi / 6$ & 2.17596 & 2.36828 & 2.43820 & 2.43383 \\
\hline$\pi / 4$ & 2.19565 & 2.56707 & 2.92311 & 3.13019 \\
\hline$\pi / 3$ & 2.21585 & 2.84882 & 4.05982 & 5.77795 \\
\hline$\pi / 2$ & 2.23651 & 3.27837 & 9.92299 & 9.50025 \\
\hline
\end{tabular}

Table 3 Critical Grashof number for primary flow $\left(G r_{c x}\right)$ for $M^{2}=10, \theta=\pi / 4, \omega=0.4$, with $K^{2}$ and $\omega T$ variation.

\begin{tabular}{|c|c|c|c|c|}
\hline$K^{2} \rightarrow$ & 4.0 & 6.0 & 8.0 & 10.0 \\
\hline$\omega T \downarrow$ & $\mathbf{G r}_{\mathbf{c x}}$ & $\mathbf{G r}_{\mathbf{c x}}$ & $\mathbf{G r}_{\mathbf{c x}}$ & $\mathbf{G r}_{\mathbf{c x}}$ \\
\hline 0 & 1.38988 & 1.25088 & 1.17166 & 1.12694 \\
\hline$\pi / 6$ & 1.39747 & 1.25291 & 1.172057 & 1.12696 \\
\hline$\pi / 4$ & 1.40331 & 1.25443 & 1.17234 & 1.12697 \\
\hline$\pi / 3$ & 1.41406 & 1.25715 & 1.17283 & 1.12698 \\
\hline $5 \pi / 12$ & 1.44809 & 1.26500 & 1.17408 & 1.12693 \\
\hline
\end{tabular}


Table 4 Critical Grashof number for secondary flow $\left(G r_{c v}\right)$ for $M^{2}=10, \theta=\pi / 4, \omega=0.4$, with $K^{2}$ and $\omega T$ variation.

\begin{tabular}{|c|c|c|c|c|}
\hline$K^{2} \rightarrow$ & 4.0 & 6.0 & 8.0 & 10.0 \\
\hline$\omega T \downarrow$ & $\mathbf{G r}_{\mathbf{c y}}$ & $\mathbf{G r}_{\mathbf{c y}}$ & $\mathbf{G r}_{\mathbf{c y}}$ & $\mathbf{G r}_{\mathbf{c y}}$ \\
\hline 0 & 3.42504 & 2.32074 & 1.88616 & 1.67069 \\
\hline$\pi / 6$ & 4.01867 & 2.41911 & 1.91810 & 1.68507 \\
\hline$\pi / 4$ & 4.68019 & 2.50081 & 1.94296 & 1.69599 \\
\hline$\pi / 3$ & 6.94456 & 2.66673 & 1.98932 & 1.71574 \\
\hline $5 \pi / 12$ & 7.74044 & 3.37511 & 2.14116 & 1.77532 \\
\hline
\end{tabular}

Table 5 Critical Grashof number for primary flow $\left(G r_{c x}\right)$ for $M^{2}=10, K^{2}=5, \omega=0.4$, with $\omega T$ and $\theta$ variation.

\begin{tabular}{|c|c|c|c|c|c|}
\hline$\omega T \rightarrow$ & 0 & $\pi / 6$ & $\pi / 4$ & $\pi / 3$ & $5 \pi / 12$ \\
\hline$\theta \downarrow$ & $\mathbf{G r}_{\mathbf{c x}}$ & $\mathbf{G r}_{\mathbf{c x}}$ & $\mathbf{G r}_{\mathbf{c x}}$ & $\mathbf{G r}_{\mathbf{c x}}$ & $\mathbf{G r}_{\mathbf{c x}}$ \\
\hline 0 & 1.26155 & 1.26519 & 1.26797 & 1.27298 & 1.28816 \\
\hline$\pi / 6$ & 1.28332 & 1.28714 & 1.29005 & 1.29531 & 1.31123 \\
\hline$\pi / 4$ & 1.31147 & 1.31553 & 1.31861 & 1.32418 & 1.34107 \\
\hline$\pi / 3$ & 1.34971 & 1.35411 & 1.35746 & 1.36350 & 1.38182 \\
\hline$\pi / 2$ & 1.40536 & 1.41033 & 1.41411 & 1.42096 & 1.44176 \\
\hline
\end{tabular}

Table 6 Critical Grashof number for secondary flow $\left(G r_{c y}\right)$ for $M^{2}=10, K^{2}=5, \omega=0.4$, with $\omega T$ and $\theta$ variation.

\begin{tabular}{|c|c|c|c|c|c|}
\hline$\omega T \rightarrow$ & 0 & $\pi / 6$ & $\pi / 4$ & $\pi / 3$ & $5 \pi / 12$ \\
\hline$\theta \downarrow$ & $\mathbf{G r}_{\mathrm{cy}}$ & $\mathbf{G r}_{\mathrm{cy}}$ & $\mathbf{G r}_{\mathrm{cy}}$ & $\mathbf{G r}_{\mathrm{cy}}$ & $\mathbf{G r}_{\mathrm{cy}}$ \\
\hline 0 & 1.97035 & 2.04568 & 2.10984 & 2.24462 & 2.90057 \\
\hline$\pi / 6$ & 2.22388 & 2.33654 & 2.43383 & 2.64266 & 3.74946 \\
\hline$\pi / 4$ & 2.72710 & 2.93904 & 3.13019 & 3.56813 & 6.76544 \\
\hline$\pi / 3$ & 4.23919 & 4.98221 & 5.77795 & 8.26952 & 18.23172 \\
\hline$\pi / 2$ & 16.62148 & 16.2931 & 9.50026 & 5.29162 & 2.08156 \\
\hline
\end{tabular}

Inspection of Table 1 shows that $G r_{c x}$ remains positive for any combination of $M^{2}$ and $\theta$. Positive Grashof number $\left(G r_{c x}>0\right)$ in free convection flows implies cooling of the plate by free convection currents i.e. transport of thermal energy from the channel plates, to the intercalated fluid. We note that the opposite case, $G r_{c x}<0$ would correspond to plate heating, wherein free convection currents transport thermal energy from the fluid to the plates. For any inclination of the magnetic field, $\theta, G r_{c x}$ magnitudes are found to increase steadily with an increase in $M^{2}$. Increasing magnetic field, $B_{0}$, as manifested by a rise in Hartmann number squared ( $M^{2}=B_{0}{ }^{2} L^{2}\left(\frac{\sigma}{\rho v}\right)^{1 / 2}$ and for constant semi-channel depth, electrical conductivity, fluid density and fluid kinematic viscosity, $M^{2}$ is directly proportional to $B_{0}{ }^{2}$ ) will cause a rise in thermal energy dissipated as a result of extra work exerted in dragging the fluid against the magnetic field. As a result greater temperatures in the fluid will be elevated and the plates will be cooled, which accounts for the increase in positive $G r_{c x}$. For low magnetic field, $\left(M^{2}=2\right) G r_{c x}$ values continuously decrease as $\theta$ increases from 0 (transverse field case) through $\pi / 6, \pi / 4, \pi / 3$ to the maximum inclination of $\pi / 2$. In the last of these cases the applied magnetic field, $B_{0}$, will be aligned with the positive $x$-direction, so Lorentzian drag will vanish from the primary (x-direction) momentum Eq. (16) as the magnetic drag term, $-M^{2} u \cos ^{2} \theta \rightarrow 0$ as $\theta \rightarrow \pi / 2$ ). Therefore since the least magnetic drag is experienced for the maximum magnetic field inclination, the least thermal energy will be dissipated by work done in dragging the fluid against the magnetic field: less heat will be convected to the plates and the $G r_{c x}$ value will be minimized $\left(G r_{c x}=\right.$ 1.07117 for $\theta=\pi / 2$ with $M^{2}=2.0$ ). With increasing magnetic field values i.e. increasing Hartmann numbers squared $\left(M^{2}=5.0 .8 .0,10.0\right)$ however there is a contrary response to that for $M^{2}=2.0 . G r_{c x}$ values are continuously increased in magnitude as $\theta$ increases from 0 through $\pi / 6, \pi / 4, \pi / 3$ to $\pi / 2$. An interesting pattern is observed also (in Table 2) for the variation of the critical Grashof number for the secondary flow field, i.e. $G r_{c y}$. In this case we observe that at any value of $M^{2}, G r_{c y}$ is continuously boosted in magnitude with a rise in magnetic field inclination i.e. secondary critical Grashof number values progressively increase as the magnetic field sweeps from the positive $z$-axis $(\theta=0)$ through to the positive $x$-axis $(\theta=\pi / 2)$. Magnitudes are also found to be considerably higher than in the case of the primary critical Grashof number $\left(G r_{c x}\right)$, particularly at high $M^{2}$ value and greater inclinations. For example at $M^{2}=8$ and $\theta=\pi / 2, G r_{c y}$ attains a value of 9.92299, and at $M^{2}=10$ and $\theta=\pi / 2, G r_{c y}$ attains the value of 9.50025 . The corresponding values of $G r_{c x}$ for these two cases are much lower and equal to 1.33969 and 1.41411 , respectively. Therefore plate cooling is much greater in the secondary flow than in the primary flow. 
In Tables 3 and 4, the evolution of the primary flow and secondary flow critical Grashof numbers, i.e. $G r_{c x}$ and $G r_{c y}$, are shown, for combinations of the rotational parameter $\left(K^{2}\right)$ and the oscillation parameter $(\omega T)$. Both tables correspond to the oblique magnetic field case $(\theta=$ $\pi / 4)$. Inspection of Table 3 reveals that with an increase in $K^{2}$ from 4, through 6, 8 to 10 , at a given $\omega T$, there is a clear decrease in $G r_{c x}$. Increasing $K^{2}$ corresponds to a rise in the rotational velocity of the channel, for fixed $L$ and $v$ ( $K^{2}=\frac{\Omega L^{2}}{v}$ ). Coriolis force is therefore boosted as $K^{2}$ is increased and this serves to reduce critical primary Grashof number magnitudes. Conversely for a fixed $K^{2}$, as the parameter $\omega T$ increases from 0 (transverse field case) through $\pi / 6, \pi / 4, \pi / 3$ to the maximum inclination of $\pi / 2$, there is a steady rise in value of Grcx. Since $\omega=0.4$, an increase in $\omega \mathrm{T}$ implies that time $(\mathrm{T})$ is increasing.

Hence with progression of time, the critical primary Grashof number is enhanced. Table 4 shows that critical Grashof number for the secondary flow $\left(G r_{c y}\right)$ exhibits a similar response to $K^{2}$ and $\omega T$ i.e. with increasing $K^{2}$ values, $G r_{c y}$ magnitudes are depressed, and with increasing $\omega T$ values, magnitudes are increased. However the values of $G r_{c y}$ are much higher than $G r_{c x}$ indicating that the secondary flow response is much stronger.

Tables 5 and 6 show the combined effects of $\omega T$ and $\theta$ on $G r_{c x}$ and $G r_{c y}$. $G r_{c x}$ as seen in Table 5, again increases with an increase in $\omega T$, for any value of magnetic field inclination $(\theta)$. However with greater $\theta$, the magnitudes of $G r_{c x}$ are clearly enhanced. Maximum $G r_{c x}$ arises therefore for the maximum studied value of $\omega T(=5 \pi / 12)$ and the maximum value of $\theta(=\pi / 2)$, and attains a value of 1.44176. A similar trend is observed for the Grcy values in Table 6. However Grcy values generally increase with increasing $\omega \mathrm{T}$ for $\theta=0, \pi / 6$ and $\pi / 4$; however as $\theta$ is further increased to $\pi / 3$ and $\pi / 2$, Grcy values rise as $\omega T$ increases to $\pi / 3$ but then fall considerably for $\omega T=\pi / 2$.

Figures 2 to 10 show the primary $(u)$ and secondary $(v)$ velocity distributions across the channel for various values of the governing parameters $\left(M^{2}, K^{2}, \theta, G r\right)$ for steady state $(\omega T=0)$ and transient $(\omega T=\pi / 4)$ cases, respectively.

Figures 2 and 3 show the effect of the rotational parameter $\left(K^{2}\right)$ on $u$ and $v$ distributions. In the steady state case (Fig. 2) with increasing $K^{2}$ primary velocity is generally reduced in magnitude. $u$ values are negative near the lower plate of the channel $(\eta=-1)$; however for the majority of the channel space values are positive so that back flow does not arise here. Peak primary velocity arises near to the upper plate and with increasing Coriolis force (i.e. greater $K^{2}$ ), the peaks are displaced further from the upper plate $(\eta=1)$. Conversely the secondary flow is found to be positive near the lower plate but strongly negative everywhere else in the channel indicating that there is strong secondary flow reversal. With increasing $K^{2}$ values, $v$ values are increased i.e. become more positive. A similar response is observed in Fig. 3 for the transient case $(\omega T=\pi / 4)$.

Figures 4 and 5 depict the primary and secondary velocity response to various Grashof numbers. Again very similar responses are observed for the steady state
(Fig. 4) and transient (Fig. 5) cases. With increasing $G r$ values, primary velocity is decelerated in the lower channel half $(-1<\eta<0)$ whereas it is strongly accelerated in the upper channel half $(0<\eta<1)$. Conversely secondary velocity is found to be strongly accelerated in the lower channel half $(-1<\eta<0)$ but decelerated in the upper channel half $(0<\eta<1)$ with an increase in $\mathrm{Gr}$ from 2 through 4, 6, 8 to 10. Primary and secondary backflow (negative $u$ and $v$ values) therefore arise generally in opposite halves of the channel. We note that primary velocity magnitudes in either half of the channel are however greater than the secondary velocity magnitudes, for any Grashof number.

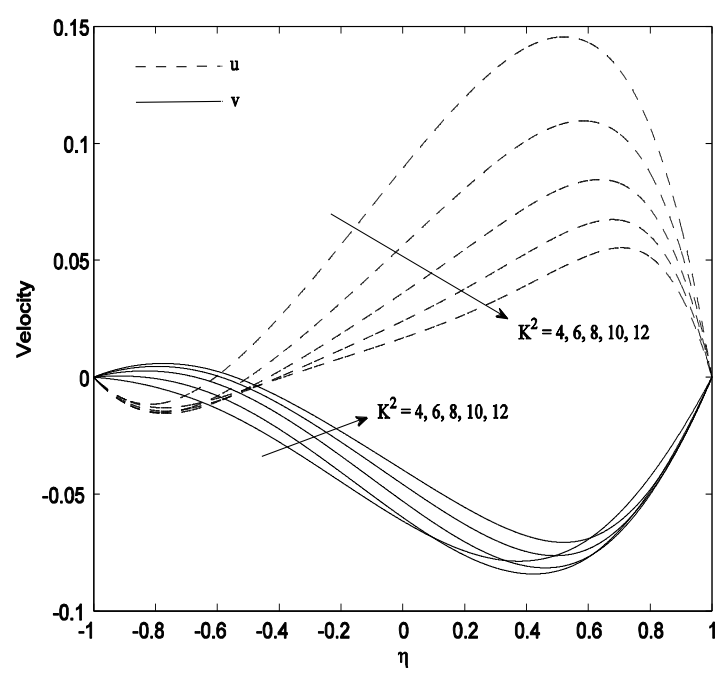

Fig. 2. Velocity distributions for $G r=2, M^{2}=10, \omega T=$ $0, \omega=0.4, \theta=\pi / 4$ for various $\mathrm{K}^{2}$ values. (Steady)

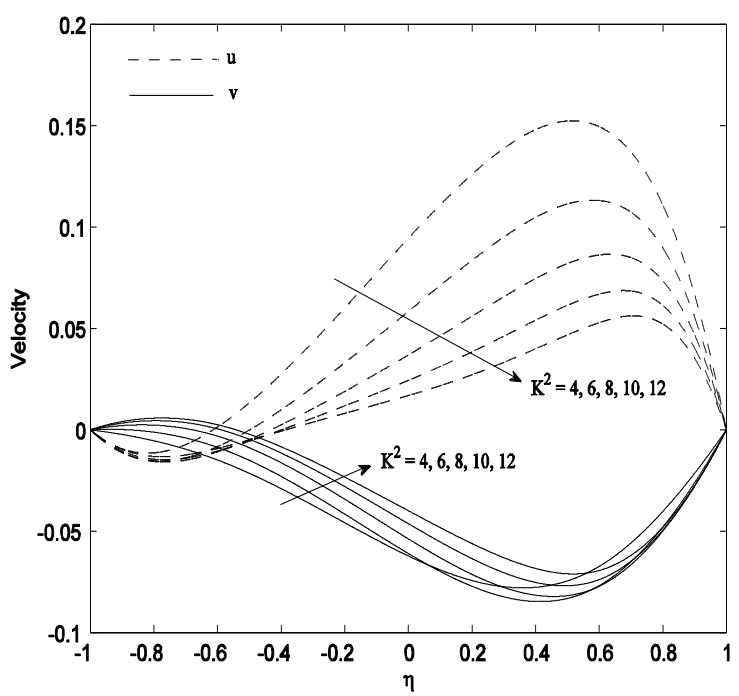

Fig. 3. Velocity distributions for $\mathrm{Gr}=2, \mathrm{M}^{2}=10, \omega \mathrm{T}=$ $\pi / 4, \omega=0.4, \theta=\pi / 4$ for various $\mathrm{K}^{2}$ values.(transient) 


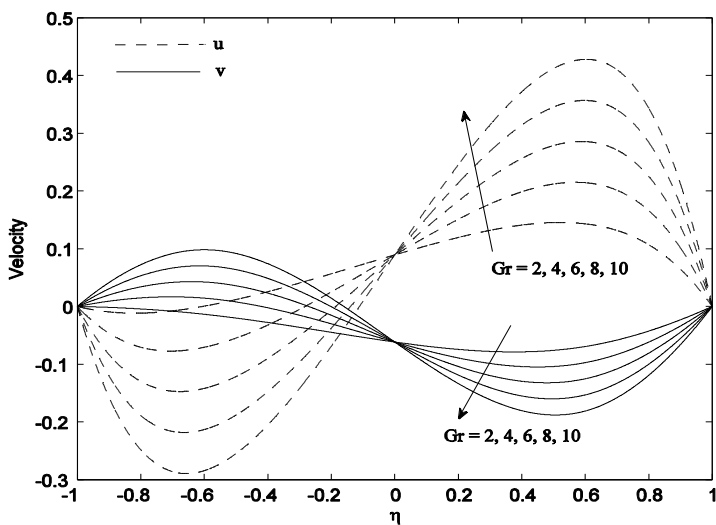

Fig. 4. Velocity distributions for $\mathrm{K}^{2}=4, \mathrm{M}^{2}=10, \omega \mathrm{T}=$ $0, \omega=0.4, \theta=\pi / 4$ for various $\mathrm{Gr}$ values.(steady)

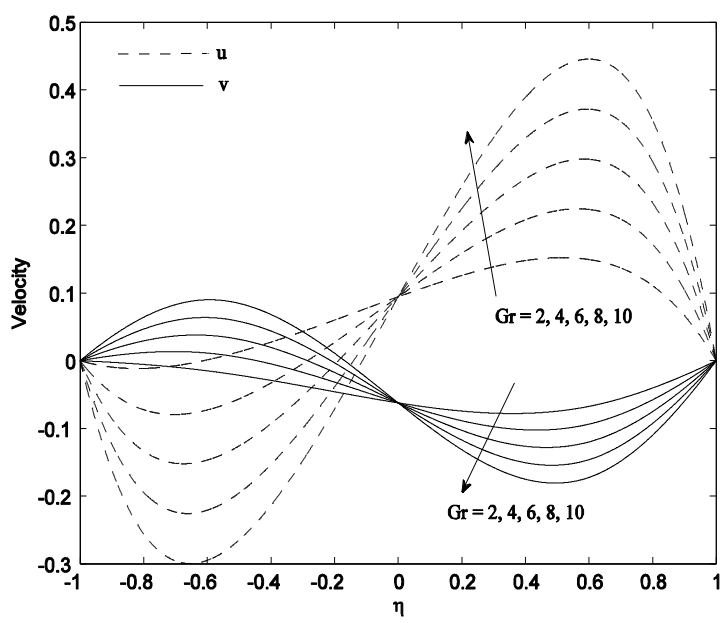

Fig. 5. Velocity distributions for $K^{2}=4, M^{2}=10, \omega T=$ $\pi / 4, \omega=0.4, \theta=\pi / 4$ for various $\mathrm{Gr}$ values. (Transient)

Figure 6 shows the influence of the oscillatory parameter $(\omega T)$ on the primary and secondary velocity profiles through the channel. Although primary flow reversal arises nearer the lower plate, initially, as we progress across the channel, with increasing $\omega T$ values (and therefore with progression of time, since $\omega$ is constrained at 0.4 ), there is a distinct acceleration in the primary flow. Conversely the secondary flow is initially decelerated with increasing $\omega T$ values as we move from the lower channel towards the channel mid-point; thereafter however the secondary velocity is slightly accelerated with increasing $\omega T$ i.e. values become less negative. Generally however the secondary velocities are negative i.e. strong secondary back flow occurs throughout the channel for all $\omega T$.

The effects of magnetic field inclination, $\theta$, on primary $(u)$ and secondary $(v)$ velocity profiles in the channel are shown in Fig. 7 and Fig. 8, again for the steady state and transient cases. The spatial distributions are very similar for both cases. For $\theta=0$, the magnetic field is in the positive z-direction (transverse magnetic field case) and for $\theta=\pi / 2$, the magnetic field is orientated along the positive $x$-direction i.e. parallel to the plane of the channel plates. As $\theta$, increases from 0 through $\pi / 6, \pi / 4$, $\pi / 3$ to $\pi / 2$, the hydromagnetic drag force,
$-M^{2} u \cos ^{2} \theta$ in the primary momentum Eq. (16), will decrease from a maximum (for $\theta=0$ for which $\cos \theta=1$ ) to a minimum (for $\theta=\pi / 2$ for which $\cos \pi / 2=0$ ). Via coupling of the primary Eq. (16) to the secondary equation (with the rotational term, $-K^{2} v$ ) and coupling of the secondary momentum Eq. (17) with the primary equation (via the rotational term, $K^{2} u$ ), the magnetic term in Eq. (16) will affect the secondary flow. In consistency with this, we observe that a rise in inclination clearly accelerates the primary flow (since hydromagnetic drag is reduced) and increase backflow in the secondary flow.

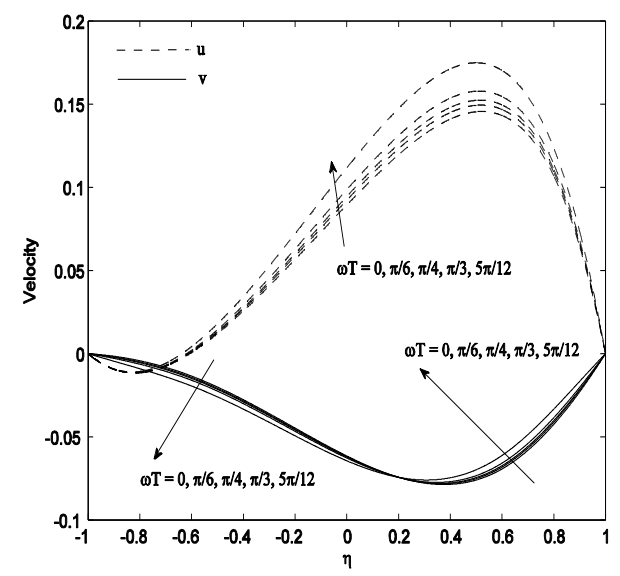

Fig. 6. Velocity distributions for $\mathrm{K}^{2}=4, \mathrm{Gr}=2, \mathrm{M}^{2}=$ $10, \omega=0.4, \theta=\pi / 4$ for various $\omega \mathrm{T}$ values.

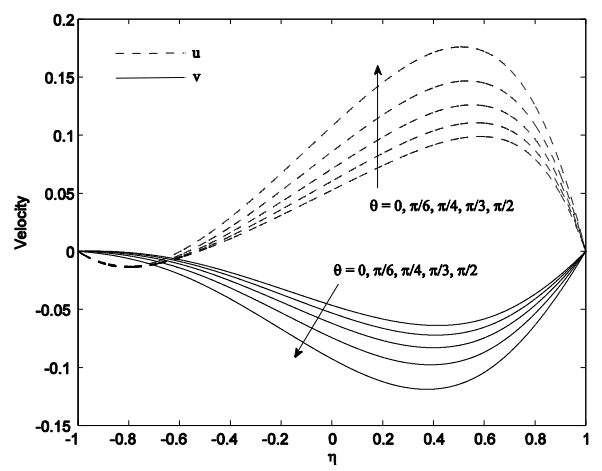

Fig. 7. Velocity distributions for $K^{2}=5, G r=2, M^{2}=$ 10, $\omega T=0, \omega=0.4$ for various $\theta$ values. (steady)

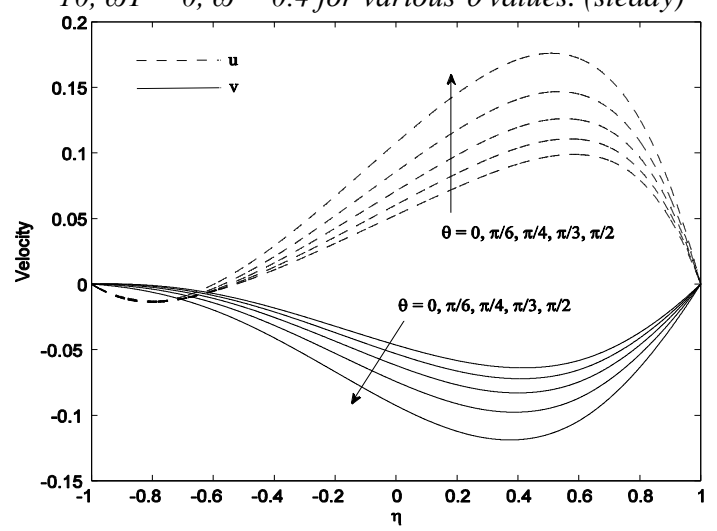

Fig. 8. Velocity distributions for $\mathrm{K}^{2}=5, \mathrm{Gr}=2, \mathrm{M}^{2}=10$, $\omega \mathrm{T}=\pi / 4, \omega=0.4$ for various $\theta$ values. (transient) 


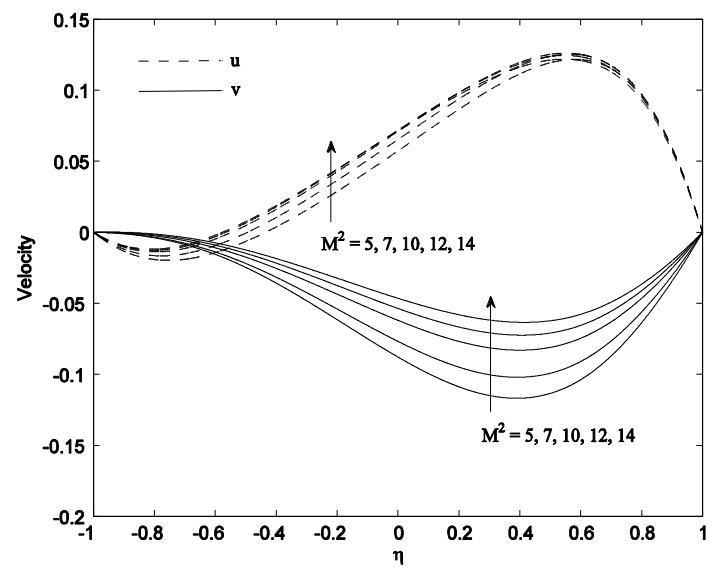

Fig. 9. Velocity distributions for $\mathrm{Gr}=2, \mathrm{~K}^{2}=5, \omega \mathrm{T}=0$, $\omega=0.4, \theta=\pi / 4$ for various $\mathrm{M}^{2}$ values. (steady)

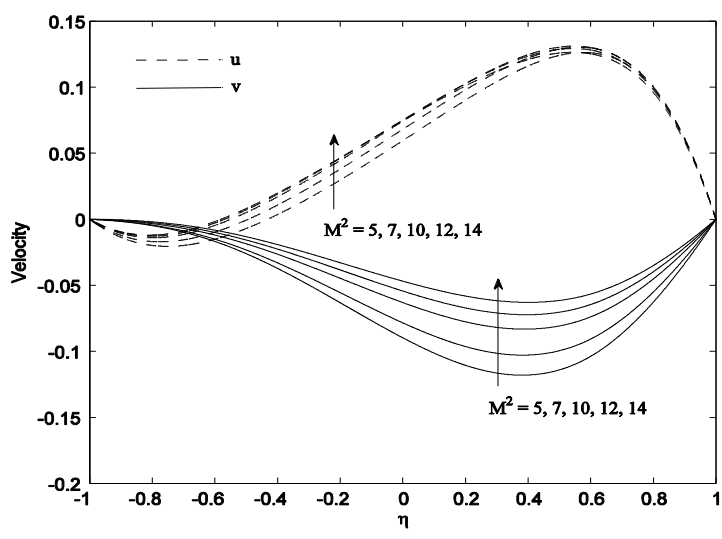

Fig. 10. Velocity distributions for $G r=2, K^{2}=5, \omega T=$ $\pi / 4, \omega=0.4, \theta=\pi / 4$ for various $\mathrm{M}^{2}$ values. (transient)

Finally in Fig. 9 and Fig. 10, we observe that for both steady state and transient cases, with an increase in the hydromagnetic parameter $\left(M^{2}\right)$, primary velocity magnitudes are decreased in magnitude in the proximity of the lower plate (where back flow arises) but increased in the remainder of the channel. Conversely secondary flow velocity magnitudes are strongly decreased throughout the channel regime with an increase in $M^{2}$ from 5, through 7,10,12 to 14 . The strong influence of magnetic field on the flow is therefore clearly visible.

\section{Conclusion}

Analytical solutions have been obtained for the transient magneto-hydrodynamic free convection flow in a rotating parallel plate channel in the presence of an inclined magnetic field. Critical Grashod numbers have been derived for the primary (main) flow and the secondary (cross) flow. The results have indicated that generally

(i) For any inclination of the magnetic field, $\theta$, Grcx and Grcy magnitudes are found to increase steadily with an increase in magnetic field parameter $\left(M^{2}\right)$.

(ii) Grcx and Grcy are increased with a rise in magnetic field inclination $(\theta)$. (iii) Grcy magnitudes are found to be substantially greater than Grcx, magnitudes, in particular at high $M^{2}$ value and greater inclination $(\theta)$.

(iv) With an increase in rotational parameter $\left(K^{2}\right)$, Grcx and Grcy are generally decreased.

(v) With increasing oscillatory parameter values $(\omega \mathrm{T})$ Grcx and Grcy magnitudes are increased.

(vi) With increasing rotational parameter $\left(K^{2}\right)$ primary velocity $(\mathrm{u})$ is decreased whereas secondary velocity $(v)$ is enhanced.

(vii) With increasing free convection parameter i.e. Grashof number (Gr) primary velocity is decelerated in the lower channel half $(-1<\eta<0)$ whereas it is strongly accelerated in the upper channel half $(0<\eta<1)$; the converse response is computed for the secondary flow.

(viii) With increasing $\omega \mathrm{T}$ values (and therefore with progression of time) there is a strong acceleration in the primary flow, whereas there secondary flow is generally retarded i.e. greater backflow.

(ix) With a rise in magnetic field inclination $(\theta)$ the primary flow is accelerated (since hydromagnetic drag is reduced) whereas the backflow is generally decelerated i.e. increasing secondary back flow.

The present model has been restricted to Newtonian flow. Future investigations will consider non-Newtonian fluids which are of interest in working operations in MHD energy generators. Furthermore the analytical solutions presented offer a good benchmark for future numerical simulations.

\section{AKNOWLEGMENTS}

The authors are thankful to the referee for valuable suggestions towards the improvement of the paper.

\section{REFERENCES}

Bég, O.A., A.Y.Bakier, V.R. Prasad, and S.K. Ghosh (2009). Nonsimilar, laminar, steady, electricallyconducting forced convection liquid metal boundary layer flow with induced magnetic field effects. Int. J. Thermal Sciences 48, 1596-1606.

Bég, O. Anwar, and S.K. Ghosh (2010). Analytical study of magnetohydrodynamic radiation-convection with surface temperature oscillation and secondary flow effects. Int. J. Applied Mathematics and Mechanics $6,1-22$.

Bég, O. Anwar, L. Sim, J. Zueco, and R. Bhargava (2010). Numerical study of magnetohydrodynamic viscous plasma flow in rotating porous media with Hall currents and inclined magnetic field influence. Communications in Nonlinear Science and Numerical Simulation J 15, 345-359.

Bég, O. Anwar, J. Zueco and L. M. López-Ochoa (2011). Network numerical analysis of opticallythick hydromagnetic slip flow from a porous spinning disk with radiation flux, variable thermophysical properties and surface injection effects. Chemical Engineering Communications 198, 360-384. 
Bég, O. Anwar, S.K. Ghosh and T. A. Bég (2011). Applied Magneto-Fluid Dynamics: Modelling and Computation, 463 pages, Lambert Academic Publishing, Saarbrucken, Germany. ISBN 978-38465-0865-7.

Bhargava, R., R. Sharma and O. Anwar Bég (2009). Oscillatory chemically-reacting MHD free convection heat and mass transfer in a porous medium with Soret and Dufour effects: finite element modeling. Int. J. Applied Mathematics and Mechanics 5 (6), 15-37.

Elco, R.A., W.F.Hughes, and F.J.Young (1962). Theoretical analysis of the radial field vortex magneto-gas dynamic generator. Zeitschrift für Angewandte Mathematik und Physik (ZAMP) 13, $1-13$.

Ferreira, J.L., S. F. da Silva, and I. da S. R^ego (2004). A multi magnetic mirror machine for plasma production with electron cyclotron resonance. Revista de F'ýsica Aplicada e Instrumentac 17, 1020.

Frank, A.M., S. R. Thomas, B. S. Denhoy, and A. K. Chargin (1977). Laser start-up system for magnetic mirror fusion Appl. Opt., 16, 1243-1252.

Ghosh, S.K. (1991). A note on steady and unsteady hydromagnetic flow in a rotating channel in the presence of inclined magnetic field. Int. J. Engineering Science 29, 1013-1016.

Ghosh, S.K. (1996). Hydromagnetic flow in a rotating channel permeated by an inclined magnetic field in the presence of an oscillator. Czech. J. Physics 46, 85-95.

Ghosh, S.K. (1997). A note on unsteady hydromagnetic flow in a rotating channel permeated by an inclined magnetic field in the presence of an oscillator. Czech. J. Physics 47, 787-792.

Ghosh, S.K. (1999). Hall effect on unsteady hydromagnetic flow in a rotating channel permeated by an inclined magnetic field in the presence of an oscillator. Czech. J. Physics 49, 465-472.

Ghosh, S.K. (2001). A note on unsteady hydromagnetic flow in a rotating channel permeated by an inclined magnetic field in the presence of an oscillator. Czech. J. Physics 51, 799-804.

Ghosh, S.K., and P.K. Bhattacharjee (2000). Magnetohydrodynamic convection flow in a rotating channel. Archives of Mechanics 52, 303318

Ghosh, S.K., and P.K. Bhattacharjee (2000). Hall effects on steady hydromagnetic flow in a rotating channel in the presence of an inclined magnetic field. Czech. J. Physics 50, 759-767.

Ghosh S.K., and I.Pop (2002). A note on a hydromagnetic flow in a slowly rotating system in the presence of an inclined magnetic field. Magnetohydrodynamics 38, 377-384.

Ghosh S.K., and I. Pop (2004). Comments on MHD plasma flow in a rotating environment in the presence of an inclined magnetic field. Int. J. Applied Mechanics and Engineering 9, 821-826.
Ghosh S.K., and I. Pop (2006). An analytical approach to MHD plasma behavior of a rotating environment in the presence of an inclined magnetic field as compared to excitation frequency. Int. J. of Applied Mechanics and Engineering 11, 845-856.

Ghosh, S.K., O. Anwar Beg, S. Rawat, T. A. Bég, and S. A. A. Ghani (2009). Rotating hydromagnetic transient convection flow of liquid metal in a porous medium with Hall and Ionslip current effects. Emirates Journal for Engineering Research 14, 1117.

Ghosh, S.K., O. Anwar Beg, and M. Narahari (2009). Hall effects on MHD flow in a rotating system with heat transfer characteristics. Meccanica 44, 741-765.

Ghosh, S.K., O.A. Bég, and J. Zueco (2009). Rotating hydromagnetic optically-thin gray gas flow with thermal radiation effects. J. Theoretical Applied Mechanics 39, 101-120.

Ghosh, S.K., O. Anwar Beg, J. Zueco, and V.R.Prasad (2010). Transient hydromagnetic flow in a rotating channel permeated by an inclined magnetic field with magnetic induction and Maxwell displacement current effects. ZAMP: J. Applied Mathematics and Physics 61, 147-169.

Hayat, T., K. Hutter, S. Nadeem, and S.Asghar (2004). Unsteady hydromagnetic rotating flow of a conducting second grade fluid. Zeitschrift für Angewandte Mathematik und Physik (ZAMP) 55, 626-641

Hayat, T., S.B.Khan, and M. Khan (2007). The influence of Hall current on the rotating oscillating flows of an Oldroyd-B fluid in a porous medium. Nonlinear Dynamics 47, 353-362.

Hughes, W., and J. Young (1966). The Electro-MagnetoDynamics of Fluids, John Wiley, New York.

Ibáñez, G., S. Cuevas, and M. López de Haro (2002). Optimization analysis of an alternate magnetohydrodynamic generator. Energy Conversion and Management 43, 1757-1771.

Kabakov, V.I., and Y.I. Yantovsky (1993). Solar pond magnetohydrodynamic generator for hydrogen production. Int. J. Hydrogen Energy 18, 19-23.

Katsurai, M. (1972). Studies on the MHD rotating machine. Electrical Engineering in Japan 92, 3143.

Kolesnikov, V. K., and V.D. Khait (1975). Nonlinear fluctuations in a MHD generator. Teplofizika Vysokikh Temperatur 13, 601-604.

Lenzo, C. S., $\quad$ P.V.Dauzvardis, and R.G. Hantman (1978). An experimental investigation of rotating-drum separators for liquid-metal MHD applications, 17th Symposium on the Engineering Aspects of Magnetohydrodynamics, Stanford, Calif., March 27-29.

Macheret, S.O., M.N.Shneider, and R.B. Miles (2004). Magnetohydrodynamic and electrohydrodynamic control of hypersonic flows of weakly ionized plasmas. AIAA J. 42, 10-19. 
Mazumder, B.S. (1977). Effect of wall conductances on hydromagnetic flow and heat transfer in a rotating channel. Acta Mechanica, 28, 85-99.

Mazumder, B.S. (1977). Effect of wall conductances on hydromagnetic flow and heat transfer in a rotating channel. Acta Mechanica, 28, 85-99.

Meyer, R.C. (1958). On reducing aerodynamic heat transfer rates by magnetohydrodynamic techniques. J. Aerospace Sci, 25, 561-566.

Michiyoshi, I., and M. Numano, (1967). Performance characteristics of a vortex-type MHD power generator - II. Plasma Physics 9, 549- 555.

Moir, R.W. (1977). Direct energy conversion in fusion reactors. Energy Technology Handbook 5.150 to 5.154 .

Naroua, H., H.S. Takhar, P.C. Ram, T. A. Bég, O. A. Bég, and R. Bhargava (2007). Transient rotating hydromagnetic partially-ionized heat-generating gas dynamic flow with Hall/Ionslip current effects: finite element analysis. Int. J. Fluid Mechanics Research 34, 6, 493-505.

Oliver, D. A. (1974). Time dependent magnetohydrodynamic generator, MIT Gas Turbine Laboratory Report, Mechanical Engineering, Massachusetts Institute of Technology, USA.

Rawat, S., R. Bhargava, Renu Bhargava, and O. Anwar Bég (2009). Transient magneto-micropolar free convection heat and mass transfer through a nonDarcy porous medium channel with variable thermal conductivity and heat source effects. Proc. IMechE Part C- J. Mechanical Engineering Science 223, 2341-2355.

Seth, G.S., and S.K.Ghosh (1986). Unsteady hydromagnetic flow in a rotating channel in the presence of inclined magnetic field. Int. J. Engineering Science 24, 7, 1183-1193.
Seth,G.S., M.S. Ansari, and R. Nandkeolyar (2010). Unsteady hydromagnetic Couette flow within porous plates in a rotating system. Adv. Appl. Math. Mech. 2, 286-302.

Sokoloff, D. (2007). Astrophysical dynamos and magnetic helicity conservation. Plasma Physics and Controlled Fusion 49, B447-B449.

Takhar, H.S., P.C. Ram, and S.S.Singh (1993). Unsteady MHD flow of a dusty viscous liquid in a rotating channel with hall currents. Int. J Energy Research 17, 69-74.

Thiagarajan, V., and V.K. Rohatgi (1977). Analysis of a collisional plasma rotating inside a cylindrical annulus. Energy Conversion 17, 73-83.

Vogin, C., and A. Alemany (2007). Analysis of the flow in a thermo-acoustic MHD generator with conducting walls. Eur. J. Mechanics - B/Fluids 26, 479-493.

Yamasaki, H., S. Kabashima, S. Shioda, and Y.Okuno (1988). Unsteady discharge and fluid flow in a closed-cycle disk MHD generator. AIAA Journal of Propulsion and Power 4, 61-67.

Yantovskiy, Y.I., and I.M. Tolmach (1963). On the theory of an asynchronous magnetohydrodynamic generator with a rotating field. Technical Report, WrightPatterson Air Force Base, Ohio, USA,

Zueco, J., O.A. Bég, H.S.Takhar, and V.R. Prasad (2009). Thermophoretic hydromagnetic dissipative heat and mass transfer with lateral mass flux, heat source, Ohmic heating and thermal conductivity effects: network simulation numerical study. Applied Thermal Engineering 29, 2808-2815.

Zueco, J., and O. Anwar Bég (2010). Network numerical analysis of hydromagnetic squeeze film flow dynamics between two parallel rotating disks with induced magnetic field effects. Tribology International 43, 532543. 